\title{
Pharmacological Activities of Compound Present in Cassia Auriculata by Pass Prediction Method
}

\author{
Article by Chandra Mohan. $\mathrm{A}^{1}$, Geetha. $\mathrm{S}^{2}$, Gajalakshmi. $\mathrm{R}^{3}$, Divya. S. $\mathrm{R}^{4}$, and Dhanarajan \\ M.S ${ }^{5}$ \\ ${ }^{1}$ Professor \\ ${ }^{2,3,4}$ Assitant Professor, PG and Research Department of Biochemistry and Chemistry, Jaya \\ College of Arts and Science, India \\ ${ }^{5}$ Registrar, Texila American University, Guyana, South America \\ E-mail: chandru2c813@gmail.com ${ }^{1}$
}

\begin{abstract}
PASS Prediction of pharmacological activity for the above compound indicated that these compounds were found to possess various pharmacological activities in the range $69.3-97.8 \%$. Both Dodecanoic Acid and n-hexadecanoic Acid were found to exhibit similar pharmacological activities as Acylcarnitine hydrolyse inhibitor (97.3\%). $\alpha$-Tocopherol exhibit the highest pharmacological activity as Lipid peroxidase inhibitor (97.8\%) among the six phytoconstituent selected for PASS prediction.
\end{abstract}

Keywords: Cassia auriculata, Phytochemical compounds, Pharmacological activity and PASS prediction.

\section{Introduction}

Cassia auriculata is one of the herbaceous plants that found throughout central and southern India, also cultivated in Punjab, Haryana, Uttar Pradesh and West Bengal. The shrub usually occurs on roadsides, waste line, and railway embankments. Avaram (Cassia auriculata Linn), family Caesalpiniaceae, is also known as Avaram tree. Cassia auriculata Linn (Family: Caesalpiniaceae) commonly known as Tanners senna, is distributed throughout hot deciduous forests of India and holds a very prestigious position in Ayurveda and Siddha systems of medicine. It was profoundly used in Ayurvedic medicine as a tonic, astringent and as a remedy for diabetes, conjunctivitis and opthalmia [1]. It is one of the principle constituents of 'Avaarai panchaga chooranam'- an Indian herbal formulation used in the treatment of diabetes to control the blood sugar level [2].

The plant has been reported to possess antipyretic [3], hepatoprotective [4], antidiabetic, antiperoxidative and antihyperglyceamic [5], microbicidal [6] and antihyperlipidaemic activities [7]. The flowers are used to treat urinary discharges, nocturnal emissions, diabetes and throat irritation [8]. They are one of the constituent of polyherbal formulation 'Diasulin' in the concentration range of 40 $\mathrm{mg} / \mathrm{dl}$ which is proven to have antidiabetic activity [9].

It has been found to possess antitumor, oncogenic, and diabeto genic properties [10]. The antioxidant and radical scavenger function of $\alpha$-tocopherol is essentially dependent on the free state of its hydroxyl group. Spectacular antiallergic and antiinflamatory activities have been attributed to DL- $\alpha$ - tocopheryl$\alpha$ - D-mannopyranoside and DL- $\alpha$-tocopheryl- $\beta$-D-galactopyranoside [11]. Hexadecanoic acid methyl ester, also known as Methyl palmitate, in the methanol fraction is an aliphatic acid ester reported to cause growth inhibition and apoptosis induction in human gastric cancer cells [12].

The phytoconstituent of a plant will often determine the physiological action on the human body. Cassia species are rich sources of Polyphenols, Anthraquinone derivatives, Flavanoids, Polysaccharides, Saponins, Tannins, and Steroids. Some of the Cassia species are rich in Glycerides with linoleic, oleic, stearic, and palmitic acids .Cassia species are well known for their laxative and purgative constituents and are also used for the treatment of skin diseases. Leaves are anthelmintic and also used to treat ulcers, skin diseases, and leprosy. An aqueous extract of leaves possesses hypoglycemic activity. The leaves are eaten as a vegetable in times of scarcity, the infusion of leaves possesses a slight purgative activity. 


\section{PASS prediction}

PASS provides simultaneous predictions of many types of biological activity based on the structure of organic compounds. It can predict more than 1500 pharmacological effects, molecular mechanism of action and toxicities on basis of structural descriptors of compounds. Thus, PASS can be used to estimate the biological activity profiles for virtual molecules, prior to their chemical synthesis and biological testing. Pa (probability to be active) estimates the chance that the studied compound is belonging to the sub-class of active compounds resembles the structures of molecules, which are the most typical in a sub-set of actives in PASS training set.

$\mathrm{Pi}$ (probability to be inactive) estimates the chance that the studied compound is belonging to the sub-class of inactive compounds resembles the structures of molecules, which are the most typical in a sub-set of inactive in PASS training set. PASS (Prediction of Activity Spectra for Substance) which is commonly used technique in drug discovery and development. PASS predict the biological activity spectrum for a compound on the basis of its structural formula [13-15].

\section{Materials and methods}

\section{Materials}

Then the plant was identified and authenticated by Plant Anatomy Research Centre (PARC/2017/3467). Phytochemical compounds present in Cassia Auriculata like Dodecanoic acid, Ethyl Caprylate, Glycine (trifluroacetyl) - methyl butyl ester, $\alpha$ - Tocopherol and $\mathrm{n}$ - Hexadecanoic acid as given in (Figure - 1 to 6) were selected for insilico prediction.

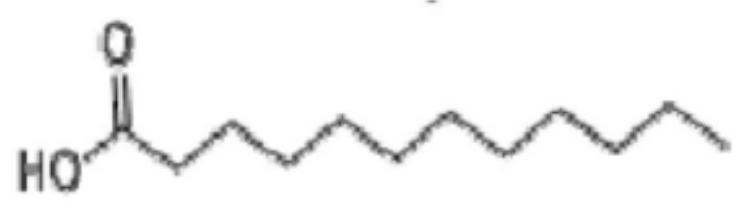

Figure 1. Dodecanoic acid

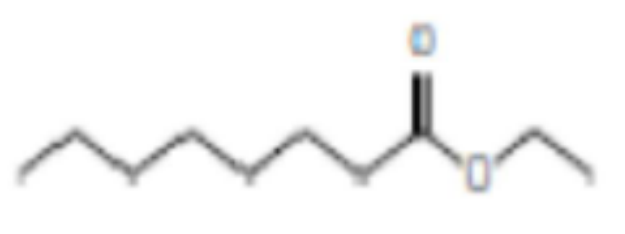

Figure 2. Ethyl caprylate

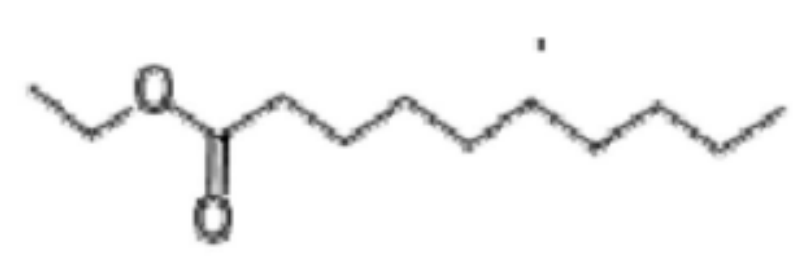

Figure 3. Capric acid ethyl ester

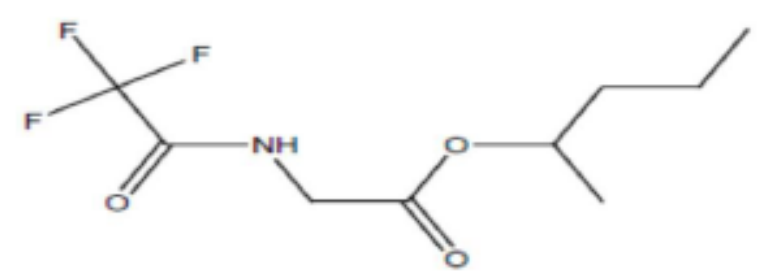

Figure 4. Glycine (trifluoroacetyl)-methyl butyl ester 


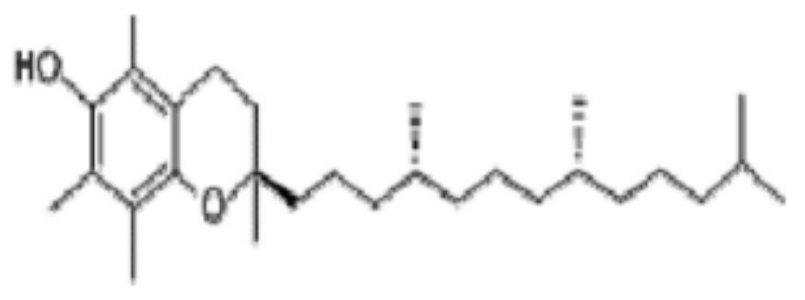

Figure 5. $\alpha$ - Tocopherol

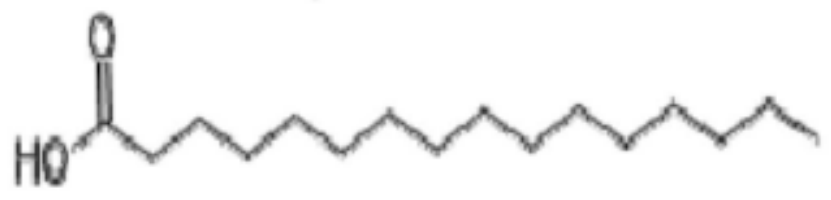

Figure 6. n-Hexadecanoic acid

\section{Methods}

\section{Pass prediction of pharmacological activity}

Various constituents of Cassia auriculata leaves extract reported were selected for predicting pharmacological activity using PASS $[16,17]$. Phytochemical compounds like a) Dodecanoic Acid, b) n-Hexadecanoic acid, c) Ethyl Caprylate, d) Capric acid ethyl ester, e) Glycine (trifluoroacetyl)-methyl butyl ester and f) $\alpha$-Tocopherol were selected. The structures of phytochemical compounds were drawn in Molinspiration online software and appear as given in (Figure-7) and their structures were saved in mol file with *.mol*.
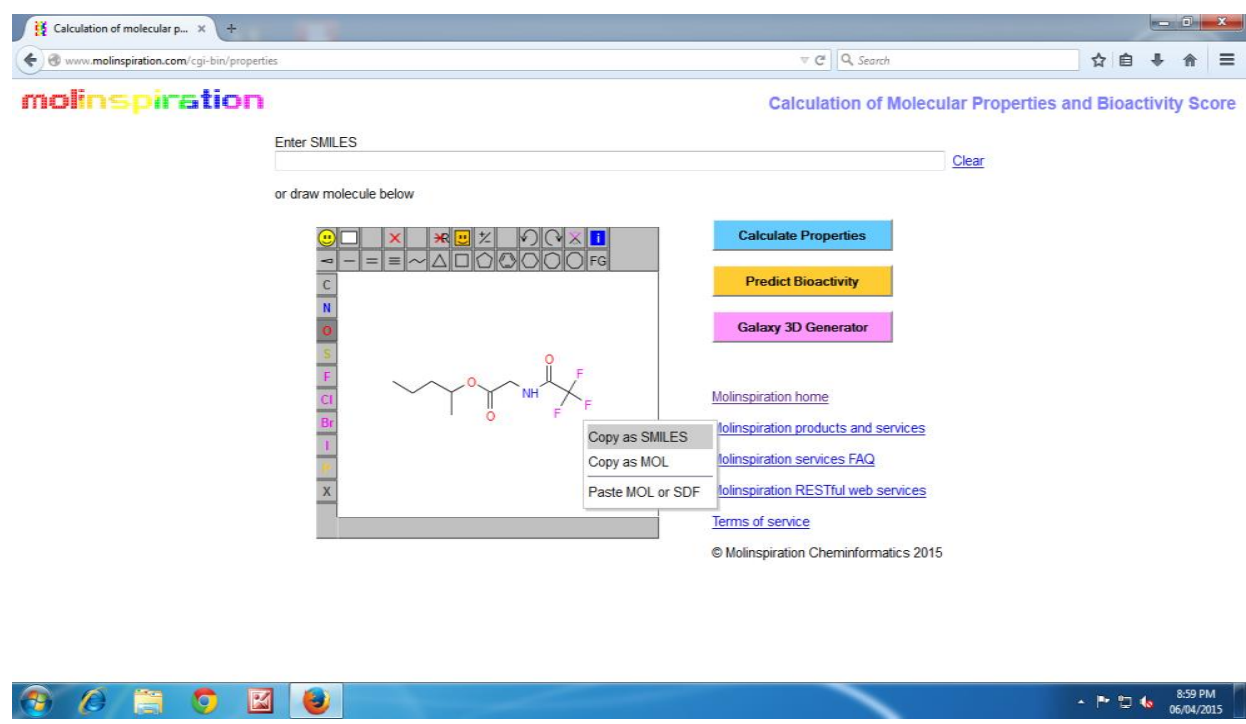

Figure 7. Molinspiration structure

PASS prediction window for prediction of pharmacological activity appeared as given in Figure-8 \& 9. 
DOI: 10.21522/TIJBMS.2016.02.02.Art004

ISSN: $2519-500 \mathrm{X}$

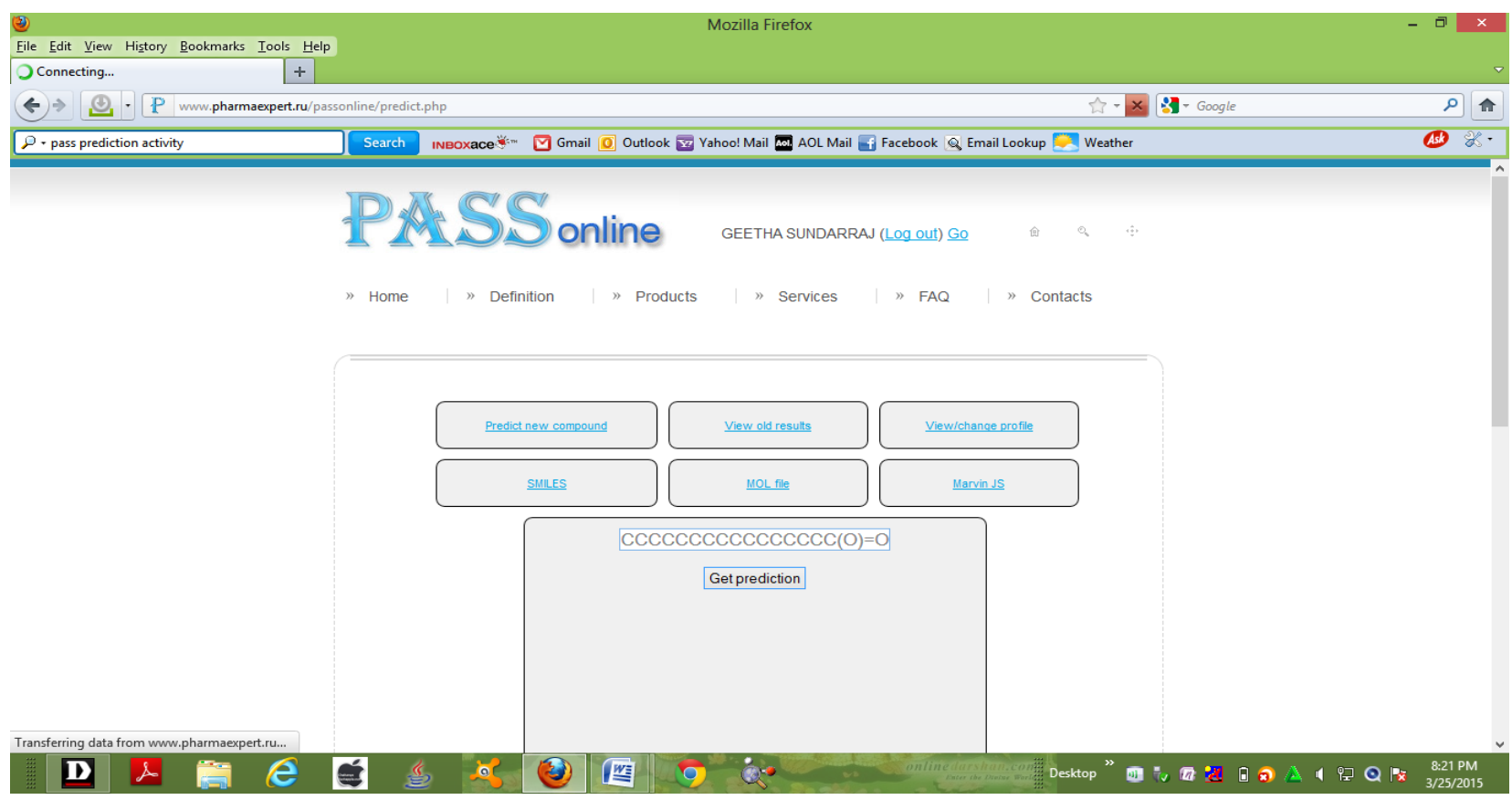

Figure 8. PASS online software setup window

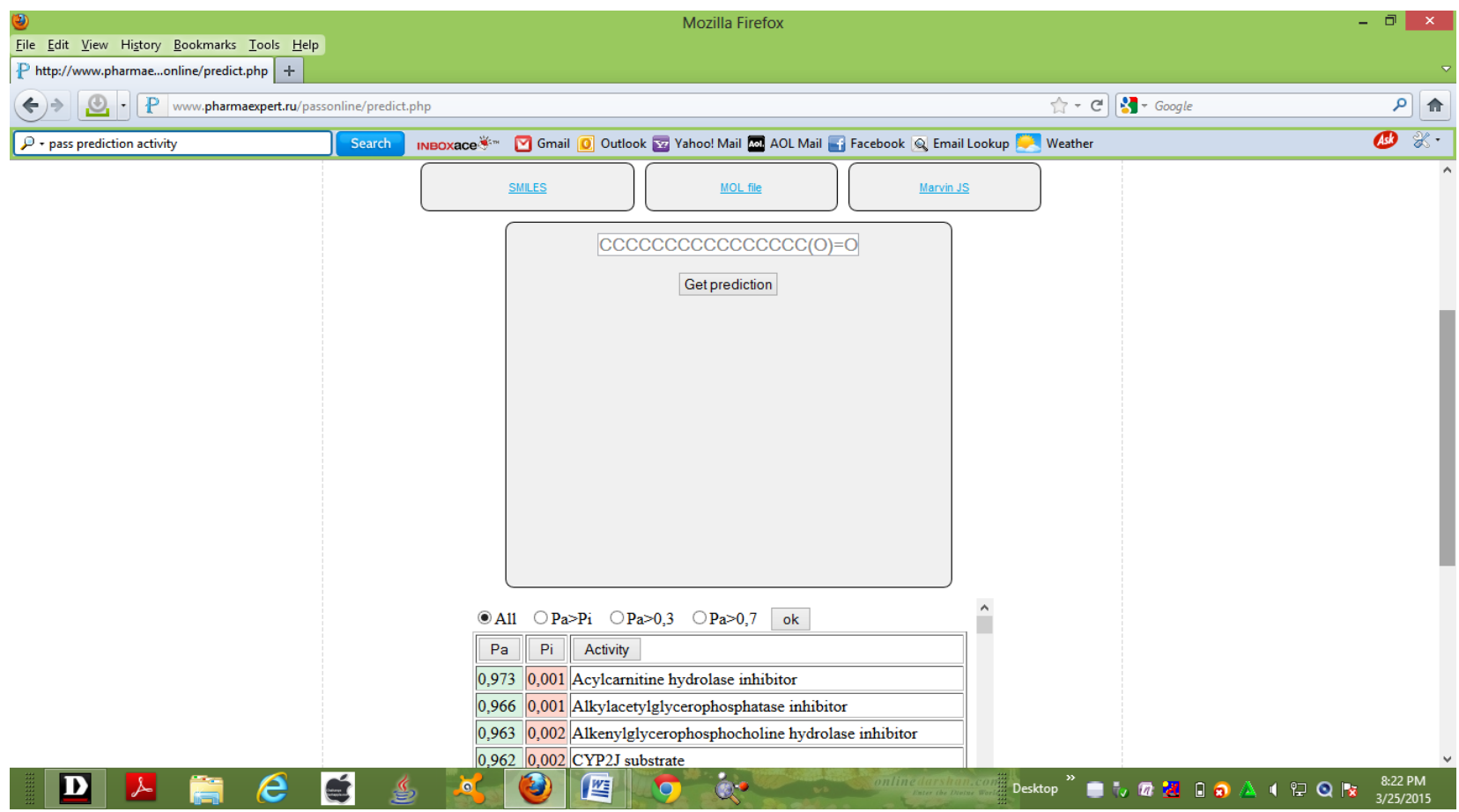

Figure 9. PASS Prediction window setup

\section{Result and discussion}

\section{PASS prediction}

All the phytochemical compounds were found to exhibit various Pharmacological activities in the range (69.3-97.8\%) as given in Table-I (a, b \& c). 
Table I (a). PASS prediction of bioactivity

\begin{tabular}{|l|l|l|l|l|}
\hline S.No & Name of the compound & Activity & $\mathbf{P}_{\mathbf{a}}$ & $\mathbf{P}_{\mathbf{i}}$ \\
\hline 1 & Dodecanoic acid & Acylcarnitine hydrolyse inhibitor & 0.973 & 0.001 \\
& & Alkylacetylglycerophosphatase inhibitor & 0.966 & 0.001 \\
& & Alknylglycerophosphocholine hydrolase & 0.963 & 0.002 \\
& & inhibitor & 0.962 & 0.002 \\
& & CYP2J substrate & 0.961 & 0.001 \\
& & CYP2J2 substrate & 0.961 & 0.002 \\
& & Acrocylindropepsin inhibitor & 0.961 & 0.002 \\
& & Chymosin inhibitor & 0.961 & 0.002 \\
& & Saccharopepsin inhibitor & 0.957 & 0.001 \\
& \multirow{5}{*}{2} & Dextranase inhibitor & 0.954 & 0.001 \\
& & CarboxypeptidaseTag inhibitor & & \\
& & All-trans-retinyl-paluitate hydrolase & 0.953 & 0.001 \\
& & inhibitor & 0.946 & 0.001 \\
& & Cutinase inhibitor & 0.934 & 0.003 \\
& & Acylcarnitine hydrolase inhibitor & 0.930 & 0.002 \\
& & Alkanal monooxygenase (FMN- linked) & 0.924 & 0.003 \\
& & inhibitor & 0.922 & 0.004 \\
& & Sugar-phosphatase inhibitor & 0.919 & 0.004 \\
& & Alkenylglycerophosphocholine & 0.919 & 0.004 \\
& & hydrolase inhibitor & 0.919 & 0.004 \\
& & Acrocylindropepsin inhibitor & 0.919 & 0.004 \\
& & Chymosin inhibitor & & \\
& & Saccharopepsin inhibitor & & \\
& & Antieczematic & & \\
& & &
\end{tabular}

Table I (b). PASS prediction of bioactivity

\begin{tabular}{|c|c|c|c|c|}
\hline 3 & $\begin{array}{l}\text { Glycine(trifluoroacetyl)- } \\
\text { methyl butyl ester }\end{array}$ & $\begin{array}{l}\text { Acrocylindropepsin inhibitor } \\
\text { Chymosin inhibitor } \\
\text { Saccharopepsin inhibitor } \\
\text { Acetylesterase inhibitor } \\
\text { Acylcarnitine hydrolase inhibitor } \\
\text { Fucosterol-epoxide lyase inhibitor } \\
\text { Pro-opiomelanocartin converting } \\
\text { enzyme inhibitor } \\
\text { Polyporopepsin inhibitor } \\
\text { Macrophage colony stimulating factor } \\
\text { agonist } \\
\text { Cutinase inhibitor }\end{array}$ & $\begin{array}{l}0.839 \\
0.839 \\
0.839 \\
0.798 \\
0.788 \\
0.745 \\
0.733 \\
0.719 \\
0.695 \\
0.693\end{array}$ & $\begin{array}{l}0.013 \\
0.013 \\
0.013 \\
0.005 \\
0.015 \\
0.011 \\
0.023 \\
0.035 \\
0.014 \\
0.010\end{array}$ \\
\hline 4 & Capric acid ethyl ester & $\begin{array}{l}\text { All-trans-retinyl-paluitate hydrolase } \\
\text { inhibitor } \\
\text { Cutinase inhibitor } \\
\text { Acylcarnitine hydrolase inhibitor } \\
\text { Alkanal monooxygenase (FMN- linked) } \\
\text { inhibitor } \\
\text { Sugar-phosphatase inhibitor } \\
\text { Alkenylglycerophosphocholine } \\
\text { hydrolase inhibitor } \\
\text { Acrocylindropepsin inhibitor } \\
\text { Chymosin inhibitor } \\
\text { Saccharopepsin inhibitor }\end{array}$ & $\begin{array}{l}0.953 \\
0.946 \\
0.934 \\
0.930 \\
0.924 \\
0.922 \\
0.919 \\
0.919 \\
0.919 \\
0.919\end{array}$ & $\begin{array}{l}0.001 \\
0.001 \\
0.003 \\
0.002 \\
0.003 \\
0.004 \\
0.004 \\
0.004 \\
0.004 \\
0.004\end{array}$ \\
\hline
\end{tabular}




\begin{tabular}{|l|l|l|l|l|}
\hline & Antieczematic & & \\
\hline
\end{tabular}

Table I (c). PASS prediction of bioactivity

\begin{tabular}{|l|l|l|l|l|}
\hline 5 & $\alpha$ - Tocopherol & Lipid peroxidase inhibitor & 0.978 & 0.002 \\
& & Peroxidase inhibitor & 0.971 & 0.001 \\
& & Antioxidant & 0.968 & 0.002 \\
& & TP53 expression inhibitor & 0.959 & 0.003 \\
& & CYP2C12 substrate & 0.955 & 0.004 \\
& & Acute neurologic disorders treatment & 0.935 & 0.004 \\
& & Antihypercholesterolemic & 0.932 & 0.003 \\
& & Antiischemic, cerebral & 0.931 & 0.005 \\
& n-Hexadecanoic acid & Reductant & 0.924 & 0.003 \\
& & AR expression inhibitor & 0.851 & 0.002 \\
\hline 6 & Acylcarnitine hydrolyse inhibitor & 0.973 & 0.001 \\
& & Alkylacetylglycerophosphatase inhibitor & 0.966 & 0.001 \\
& & Alknylglycerophosphocholine hydrolase & 0.963 & 0.002 \\
& inhibitor & 0.962 & 0.002 \\
& & CYP2J substrate & 0.961 & 0.001 \\
& & CYP2J2 substrate & 0.961 & 0.002 \\
& & Acrocylindropepsin inhibitor & 0.961 & 0.002 \\
& & Chymosin inhibitor & 0.961 & 0.002 \\
& & Saccharopepsin inhibitor & 0.957 & 0.001 \\
& & Dextranase inhibitor & 0.954 & 0.001 \\
& & CarboxypeptidaseTag inhibitor & & \\
\hline
\end{tabular}

Dodecanoic acids various pharmacological activities as given in Table - I (a) showed that this exhibited very good inhibitors as Acylcarnitine hydrolyse inhibitor (97.3\%), Alkylacetyl glycerophosphocholine hydrolyse inhibitor (96.6\%), Alknylglycerophosphocholine Ethyl caprylate was also observed to exhibit various pharmacological activities in the range $91.9-95.3 \%$ as All-transretinyl-paluitate hydrolase inhibitor (95.3\%), Cutinase inhibitor (94.6\%), Acylcarnitine hydrolase inhibitor (93.4\%), Alkanal monooxygenase (FMN- linked) inhibitor (93.0\%), Sugar-phosphatase inhibitor (92.4\%), Alkenylglycerophosphocholine hydrolase inhibitor (92.2\%), Acrocylindropepsin inhibitor (91.9\%), Chymosin inhibitor (91.9\%) and Saccharopepsin inhibitor (91.9\%) respectively.

Glycine (trifluoroacetyl) - methyl butyl ester exhibited various pharmacological acitivities as Capric acid ethyl ester exhibited various pharmacological activities as All-trans-retinyl-paluitate hydrolase inhibitor (95.3\%), Cutinase inhibitor (94.6\%), Acylcarnitine hydrolase $\alpha$ - Tocopherol was also observed to exhibit various pharmacological activities in the range $85.1-97.8 \%$ as Lipid peroxidase inhibitor (97.8\%), Peroxidase inhibitor (97.1\%), TP53 expression inhibitor (95.9\%) and AR expression inhibitor (85.1\%). n-Hexadecanoic acids various pharmacological activities as given in Table - I (c) showed that this exhibited very good inhibitors as Acylcarnitine hydrolyse inhibitor (97.3\%), Alkylacetylglycerophosphocholine hydrolyse inhibitor (96.6\%), Alknylglycerophosphocholine. hydrolase inhibitor (96.3\%), Acrocylindropepsin inhibitor (96.1\%), Chymosin inhibitor (96.1\%),Saccharopepsin inhibitor (96.1\%), Dextranase inhibitor (95.7\%) and CarboxypeptidaseTag inhibitor $(95.4 \%)$ respectively.

\section{Conclusion}

PASS Prediction of pharmacological activity for the above compound indicated that these compounds were found to possess various pharmacological activities in the range $69.3-97.8 \%$. Both Dodecanoic Acid and n-hexadecanoic Acid were found to exhibit similar pharmaceutical activities as Acylcarnitine hydrolyse inhibitor. $\alpha$-Tocopherol exhibit the highest pharmaceutical activity as Lipid peroxidase inhibitor (97.8\%) among the six phytoconstituent selected for PASS prediction. 


\section{Reference}

[1]. Anastas P.T., Levy I.J., Parent K.E. (Eds). Green Chemistry Education. Changing the Course of Chemistry, ACS Publications, Washington DC, 2009.

[2]. Basu and Kirtikar. Indian Medicinal Plants. Vol. II, Second edition .International Book distributors Dehradun India, 867-868; 1935.

[3]. Carp O., Huisman C.L., Reller A., Photoinduced reactivity of titanium dioxide. Prog in Solid State Chem, 32: 33-117; 2004.

[4]. Evans. Trease W.C. and Evans Pharmacognosy W.B. Saunders Company Ltd., London, pp (14th Edition). 19-20; 2000.

[5]. Grossman E. Chasing Molecules: Poisonous Products, Human Health, and the Promise of Green Chemistry. Island Press, New York, 2009.

[6]. Lipinski C.A. Drug Discovery Today: Technologies; 1 (4): 337-34; 2004.

[7]. Mossi A.J., Mazutti, Paroul M., Corazza N., Dariva M.L., Cansian C. \& Oliveira R.L., Rocha O.R., Dantas R.F., Duarte M.M.M.B., et al. Oil sludge treatment by photocatalysis applying black and white light. Chem Eng J, 157: 80-85; 2010.

[8]. Mukunthan K.S., Elumalai E.K., Trupti N.P., Ramachandra Murty V. Catharanthus roseus: a natural source for the synthesis of silver nanoparticles. Asian Pacific Journal of Tropical Biomedicine, 270-274; 2011.

[9]. Mor G.K., Varghese O.K., Paulose M., et al. A review on highly ordered, vertically oriented $\mathrm{TiO}_{2}$ nanotube arrays: Fabrication, material properties, and solar energy applications. Solar Energ Mater Solar Cell, 90: 20112075; 2006.

[10]. Mallikadevi S., Palulsamy T.S., Jamuna S. and Karthika K., Analysis for Phytoceuticals And Bioinformatics Approach For the Evaluation of Therapetic Properties of Whole Plant Methanolic Extract of Mukia Maderaspatana (L.) M.Roem. (Cucurbitaceae) - ATraditional Medicinal Plant in Western Districts of Tamil Nadu, India, Asian Journal of Pharmaceutical and Clinical Research, 5(4):2241; 2012.

[11]. Newman D.J., Cragg G.M., Snadder K.M. J. Nat. Prod., 66(7): 1022 -1037; 2003.

[12]. Prakash S.K... Int. J. Poultry Sci. 5: 259-261; 2006.

[13]. Sharma S.K. Green Chemistry for Environmental Sustainability. Series: Advancing Sustainability through Green Chemistry and Engineering. CRC Press, Boca Raton, FL, 2010.

[14]. Thirumurgan A., Tomy N.A., Jai Ganesh R., Gobikrishnan S.. Biological reduction of silver nanoparticles using plant leaf extracts and its effect an increased antimicrobial activity against clinically isolated organism. De. Phar. Chem, 2: 279-284; 2010;

[15]. Tripathy A., Raichur A.M., Chandrasekaran N., Prathna T.C., Mukherjee A., Nanopart. J. Res.12, 237 ; 2010. DOI: $10.1007 / \mathrm{s} 11051-009-9602-5$.

[16]. Tagboto S., Townson S., Adv. Parasitol., 50: 199-295; 2001. 